\title{
Paper-based Chemiluminescence Device with Co-Fe Nanocubes for Sensitive Detection of Caffeic Acid
}

\author{
Liu Zhang, Yue Hou, Xiaoyan Guo, Wei Liu, ${ }^{\dagger}$ Congcong Lv, Xing Peng, and Zixuan Zhang \\ Key Laboratory of Analytical Chemistry for Life Science of Shaanxi Province, School of Chemistry and \\ Chemical Engineering, Shaanxi Normal University, Xi'an 710062, P. R. China
}

\begin{abstract}
In this work, a new chemiluminescence (CL) system of Co-Fe prussian blue analogs nanocubes (Co-Fe PBA NCs) that can catalyze luminol to produce strong CL in the absence of $\mathrm{H}_{2} \mathrm{O}_{2}$ was established. Co-Fe PBA NCs have the property of oxidase-like activity, and it can catalyze the generation of active oxygen radicals in a dissolved oxygen system. Since caffeic acid (CA) can remove reactive oxygen species in the system, a sensitive detection method for CA on a paper-based chip was developed. Under the optimal conditions, this method showed a good linear response to CA in the range of $10-800 \mathrm{ng} \mathrm{mL} \mathrm{m}^{-1}$ with a limit of $3 \mathrm{ng} \mathrm{mL}^{-1}$. The proposed method had been used for the determination of CA in tea samples. The results may open a new avenue for the catalytic property on luminol CL system without extra oxidants.
\end{abstract}

Keywords Co-Fe prussian blue analogs nanocubes, chemiluminescence, luminol, caffeic acid

(Received June 27, 2020; Accepted August 21, 2020; Advance Publication Released Online by J-STAGE August 28, 2020)

\section{Introduction}

Phenolic compounds (PCs) widely exist in vegetables and fruits, as well as in beverages such as wine, tea and juice. ${ }^{1-3}$ It can reduce the destructure effect of reactive oxygen species on the human body and participate to improve the antioxidant level in human blood. ${ }^{4}$ Caffeic acid (CA), which is known as 3,4-dihydroxycinnamic acid, is one of the important PCs. Since it has the same function as PCs for preventing the occurrence of cancer, heart disease and other diseases, ${ }^{3}$ the detection of CA can be useful for ranking the antioxidant capacity of foods. In recent years, different methods such as fluorescence,${ }^{6}$ mass spectrometry, ${ }^{7}$ electrochemical, ${ }^{8}$ capillary zone electrophoresis ${ }^{9}$ and high-performance liquid chromatography ${ }^{10}$ have been developed for detecting CA. However, some of these methods are time-consuming and need expensive instruments. So, a simple and sensitive method is required for CA detection. Chemiluminescence $(\mathrm{CL})^{11-13}$ has the advantages of fast speed, simple instrumentation, wide linear range and high sensitivity. ${ }^{14,15}$ Therefore, CL analysis has been widely used in diverse fields including biology, food analysis, ${ }^{16}$ pharmacology ${ }^{17}$ and clinical assays. ${ }^{18}$ In recent years, CL has been used for the detection of PCs and the evaluation of antioxidant capacity. ${ }^{19}$ Kefalas' team studied the antioxidant activity of four kinds of antioxidants mixtures (uncliptin, ascorbic acid, catechin, CA) under different mole ratios with a luminol-Co(II)-EDTA system. ${ }^{19} \mathrm{~A} \mathrm{KMnO}_{4-}$ $\mathrm{CH}_{2} \mathrm{O}$ CL system ${ }^{20}$ and a $\mathrm{Ce}(\mathrm{IV})-\mathrm{Rh} 6 \mathrm{G} \mathrm{CL}^{21}$ system have been developed for the determination of PCs in food. A microfluidic device ${ }^{22}$ was used with a $\mathrm{NaHCO}_{3}-\mathrm{H}_{2} \mathrm{O}_{2}-\mathrm{Co}^{2+} \mathrm{CL}$ system to detect the antioxidants (gallic acid, catechin, CA, and rutin) by an inhibiting effect in honey and fruit. Although these CL

† To whom correspondence should be addressed. E-mail: weiliu@126.com, liuwei2@hotmail.com reactions were simple and these agents were easy to obtain, the sensitivity for antioxidants was still low. In 2019, Lawati's research group ${ }^{16}$ reported that a new $\mathrm{H}_{2} \mathrm{O}_{2}$-rhodamine $\mathrm{b}$ (RhoB)CoMOF CL system for PCs detection on a paper-based device. With RhoB@CoMOF loaded on paper, PCs can be sensitively detected in food samples. A new catalyst of CoMOF was adopted in this work, and developing new catalysts is a trend in CL research.

Nowadays, multi-metal nanomaterials ${ }^{23-27}$ have been reported in the CL system with better catalytic activity characteristics compared to monometallic nanomaterials. ${ }^{28,29} \mathrm{Au}-\mathrm{Ag}$ nanoparticles were prepared with an excellent catalytic property to the luminol$\mathrm{H}_{2} \mathrm{O}_{2} \quad \mathrm{CL}$ system. ${ }^{23} \mathrm{Cu} / \mathrm{Ni}$ nanoparticles ${ }^{24}$ can significantly increase the $\mathrm{Cl}$ signal of the $\mathrm{NaHCO}_{3}-\mathrm{H}_{2} \mathrm{O}_{2}$ system. Superior catalytic abilities with $\mathrm{Cu} / \mathrm{Co}$ nanorods ${ }^{25}$ and $\mathrm{CoFe}_{2} \mathrm{O}_{4}$ nanoparticles ${ }^{26}$ were also expressed in the luminol CL system. Huang's group ${ }^{27}$ has synthesized FeCo alloy@N-doped carbon layers with an excellent catalytic property in the luminol- $\mathrm{H}_{2} \mathrm{O}_{2}$ system. Fe ${ }^{\mathrm{III}}$-Co PBA nanocubes were prepared with $\mathrm{FeCl}_{3} \cdot 6 \mathrm{H}_{2} \mathrm{O}$ and $\mathrm{K}_{3}\left[\mathrm{Co}(\mathrm{CN})_{6}\right]$. Nowadays, Lou's group ${ }^{30}$ has reported an oriented assembly of $\mathrm{Co}-\mathrm{Fe}$ prussian blue analogs nanocubes (Co-Fe PBA NCs) with a simpler method. Different valence states of $\mathrm{Fe}$ and $\mathrm{Co}$ existed in the anisotropic nanoparticles. Compared to Huang's ${ }^{27}$ work, we found that this blue $\mathrm{Co}-\mathrm{Fe}$ nanocubes (Co-Fe PBA NCs) can directly catalyze the luminol to produce a strong CL intensity without $\mathrm{H}_{2} \mathrm{O}_{2}$. Since CA can inhibit the CL signal, a new CL platform was established for CA detection with high sensitivity.

Paper-based analytical devices $(\mu \mathrm{PADs})^{15}$ have gained fast progress during the past decade, owing to the intrinsic advantages of low-cost, easy-to-use, portability and disposibility. As liquid flow can be easily achieved by capillary forces, it can be easily fabricated, and has been used in many fields. ${ }^{31}$ Now the applications of $\mu$ PADs have grown exponentially in the field of point-of-care (POC) diagnosis ${ }^{31}$ as well as in the field of food 
safety. ${ }^{32}$ Thus, in this work, the $\mu$ PADs platform was used for the detection of $\mathrm{CA}$ in tea samples. Based on the inhibitory effect of CA on the luminol-Co-Fe PBA NCs CL system, CA can be sensitively detected on $\mu$ PADs with a detection limit of $3 \mathrm{ng} \mathrm{mL} \mathrm{m}^{-1}$. Impressively, the proposed platform was successfully used for the detection of CA in tea samples with high precision and good reproducibility.

\section{Experimental}

\section{Reagents and materials}

All reagents were of analytical grade. Cobalt(II) acetate tertrahydrate, CA, gallic acid, sinapic acid, coumaric acid, and superoxide dismutase (SOD) (BR, 2500-7000 u) were purchased from Alpha Chemical Reagents Co., Ltd. (Zhengzhou, China). Trisodium citrate was purchased from Chemical Reagent Factory (Xi'an, China). Iron(III) chloride hexahydrate, sodium chloride, potassium chloride, calcium chloride, copper(II) chloride dehydrate, nickel nitrate, cadmium chloride, magnesium chloride hexahydrate, and zinc acetate were purchased from Sinopharm Chemical Reagent Co., Ltd. (Shanghai, China). Ascorbic acid (AA) was purchased from Adamas Reagent Co., Ltd. (Shanghai, China). Potassium ferricyanide, 3-aminophthalhydrazide (luminol), 2,5dimethylfuran, tartaric acid, citric acidmalic, acid and thiourea were purchased from Aladdin Reagent Co., Ltd. (Shanghai, China). Ethanol and citric acid monohydrate were purchased from Sinopharm Chemical Reagent Co., Ltd. (Shanghai, China). Whatman chromatography paper \#1 (WCP\#1) (200.0 $\times$ $200.0 \mathrm{~mm}, 0.18 \mathrm{~mm}$ thick) was purchased from Sigma (USA). Millipore Milli-Q water $(18.2 \mathrm{M} \Omega \mathrm{cm})$ was used in all experiments. A $10^{-2} \mathrm{M}$ luminol stock solution was prepared and stored in the dark for one week so as to at least ensure that the reagent had stabilized.

\section{Apparatus}

The paper-based chips were made by a ColorQube 8570 wax ink printer (Fuji, Japan). The CL intensity was detected and recorded with a Model IFFS-ACL analyzer (Xi'an Remex Electronic Sci. Tech. Co., Ltd. Xi'an, China). CL data acquisition and treatment were performed by using of RFL-1 software (Xi'an Remax, Xi'an, China). A scanning electron microscope (SEM) (Hitachi, Japan) was used to characterize the morphology of the as-prepared Co-Fe PBA NCs. A X-ray diffractometer (Bruker, Germany) was employed for a powder X-ray diffraction (XRD) study. Fourier transform infrared (FT-IR) spectra were obtained in the range from 4000 to $2000 \mathrm{~cm}^{-1}$ using an a Prestige-21 FT-IR spectrometer (Bruker, Gemany) with a $\mathrm{KBr}$ pellet. UV absorption spectra were measured on a UV-1800 spectrophotometer (Shimadzu, Japan). The electron spin resonance (ESR) spectra were recorded on an Electron paramagnetic spectrometer (Bruker, Germany).

\section{Preparation of a paper-based chip using a wax ink printer}

The design was the same as our published paper $^{33}$ by a wax printing method. Firstly, the shape of the paper-based analytical device with a circle design was obtained by Coredraw X6 software. The reaction or detection zone had a diameter of $6 \mathrm{~mm}$. Then, the design was printed onto paper by a wax ink printer. After that the printed papers were placed in an oven and baked at $120^{\circ} \mathrm{C}$ for $5 \mathrm{~min}$. Wax could melt and penetrate into the paper fiber to form the hydrophobic part on the paper. Finally, the paper-based chip was cooled at room temperature and prepared for following experiments.

\section{Synthesis of Co-Fe PBA NCs}

The Co-Fe PBA NCs were prepared just as the method for typical preparation. ${ }^{30}$ The next two solutions were prepared. Firstly, $0.073 \mathrm{~g}$ of trisodium citrate and $0.075 \mathrm{~g}$ of cobalt(II) acetate tertrahydrate were dissolved in $20 \mathrm{~mL}$ of ultrapure water. Secondly, $0.066 \mathrm{~g}$ of potassium ferricyanide was dissolved in $30 \mathrm{~mL}$ of ultrapure water. Then, the upper two solutions were mixed together under stirring; 1 min later, the solution turned to a brown color and then the solution was then aged at $35^{\circ} \mathrm{C}$ for $36 \mathrm{~h}$. Finally, the mazarine precipitate was collected by centrifugation and washed with ultrapure water and ethanol four times. At last, the product was dried at room temperature to form mazarine powder.

Catalytic activity of Co-Fe PBA NCs for the luminol CL system on the paper-based chip

The next procedures were used for CL detection on the paperbased chip with Co-Fe PBA NCs. Firstly, the IFFS-A CL Analyzer was reheated for $30 \mathrm{~min}$. Then, a $2.5 \mathrm{mg} \mathrm{mL}^{-1} \mathrm{Co}-\mathrm{Fe}$ PBA NCs solution was prepared by dissolving in ultrapurhe water. The paper was put on the bottom of a dark box with a $\mathrm{CL}$ instrument. Then, $2.5 \mu \mathrm{L}$ of the Co-Fe PBA NCs solution was spotted on the paper reaction area. The dark box was then shut with a black metallic cover, and $10 \mu \mathrm{L}$ of the luminol solution was quickly injected onto the paper device by an injector through an injection hole, which was on top of the dark box. The CL signal was detected within $1-2 \mathrm{~s}$ and data acquisition was performed using RFL software running under Windows XP.

\section{Detection of CA in an aqueous medium and tea samples}

The green and black tea samples were purchased from a local market; $0.1 \mathrm{mg}$ tea samples were dissolved in $10 \mathrm{~mL}$ of distilled water. A CL analysis of CA was carried out by the following procedures: $10 \mu \mathrm{L}$ of ultrasonic-dispersed Co-Fe PBA NCs solution and $10 \mu \mathrm{L}$ of certain amounts of $\mathrm{CA}$ or tea sample were mixed in a $1.5-\mathrm{mL}$ centrifuge tube; a $2.5-\mu \mathrm{L}$ of mixture solution was then spotted on the paper-based reaction area. The paper was put on the bottom of the dark box with the CL instrument. After that, $5 \mu \mathrm{L}$ of a $1.4 \mathrm{mM}$ luminol solution was quickly injected on the paper-based reaction area (Scheme 1). The CL signals were detected and collected with RFL software running under Windows XP.

\section{Results and Discussion}

\section{Characterization of $\mathrm{Co}-\mathrm{Fe} \mathrm{PBA} \mathrm{NCs}$}

The as-prepared Co-Fe PBA NCs were characterized by scanning electronic emission (SEM), energy dispersion spectroscopy (EDS), X-ray powder diffraction (XRD), and Fourier transform infrared (FT-IR) spectra. The morphology of the Co-Fe PBA NCs was characterized by SEM (Fig. 1A). These particles had a 3D architecture with a uniform cubic geometry. An EDS analysis confirmed the presence of $\mathrm{K}$, Co, $\mathrm{Fe}, \mathrm{C}, \mathrm{O}$, and $\mathrm{N}$ in the Co-Fe PBA NCs (Fig. 1B). Co-Fe PBA NCs were measured by XRD patterns from single-crystal X-ray data (Fig. 1C). Based on XRD, relative sharp peaks at $2 \theta=18^{\circ}$, $25^{\circ}$, and $36^{\circ}$ corresponded to the characteristic crystal plane of (200), (220), (400). FT-IR spectra showed that the characteristic absorption peaks of the hydroxyl groups were at 3377, and $3629 \mathrm{~cm}^{-1}$. The $v(\mathrm{CN})$ peaks were at $2160 \mathrm{~cm}^{-1}$ for $\mathrm{Co}(\mathrm{II})-$ $\mathrm{N} \equiv \mathrm{C}-\mathrm{Fe}$ (III) and $2083 \mathrm{~cm}^{-1}$ for $\mathrm{Co}(\mathrm{II})-\mathrm{N} \equiv \mathrm{C}-\mathrm{Fe}(\mathrm{II})$. Furthermore, the emergence of a strong $v(\mathrm{CN})$ peak for $\mathrm{Co}(\mathrm{III})-\mathrm{N} \equiv \mathrm{C}-\mathrm{Fe}(\mathrm{II})$ at $2123 \mathrm{~cm}^{-1}$ was observed. FT-IR spectra indicated that there 


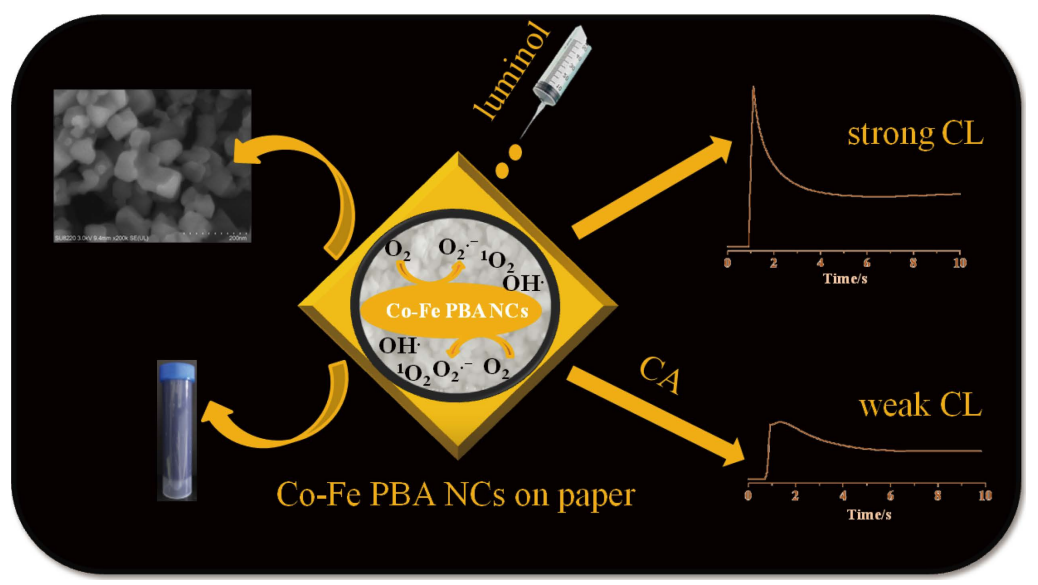

Scheme 1 The schematic diagram for the CL detection of CA with the luminol-Co-Fe PBA NCs system.

A
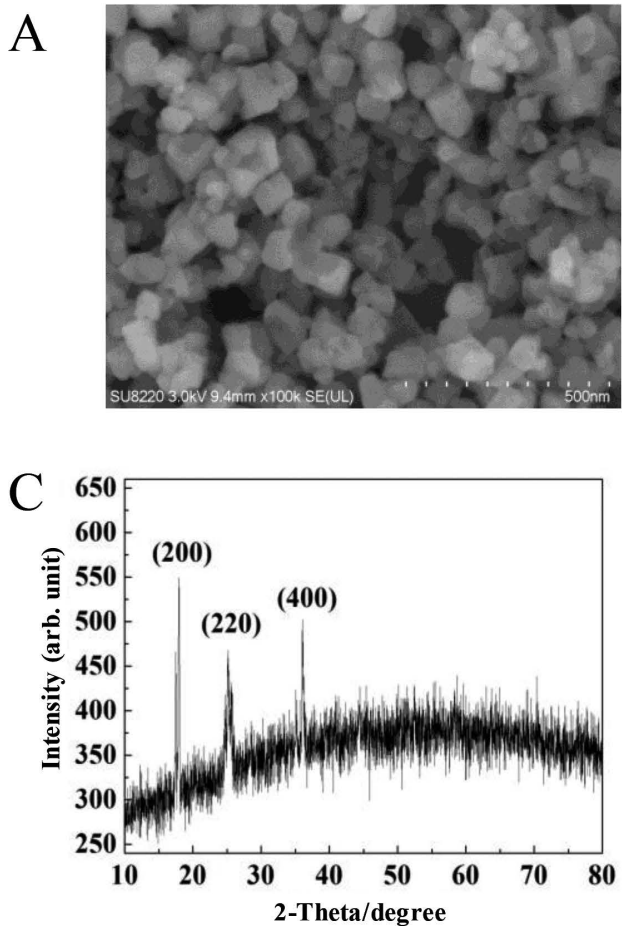

Fig. 1 Characterization of the Co-Fe PBA NCs. spectrum; (C) XRD; (D) FT-IR spectrum.

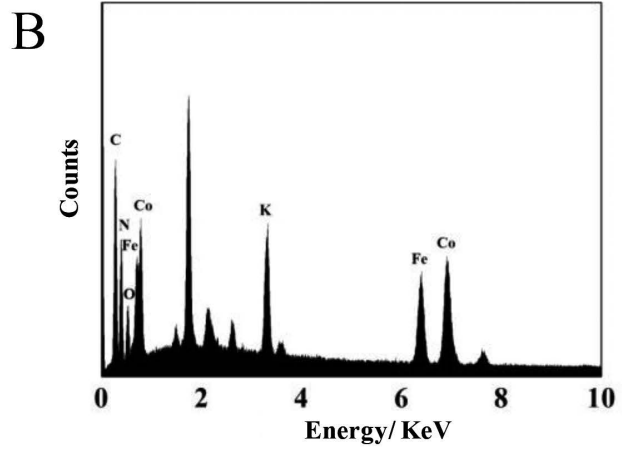

$\mathrm{D}$

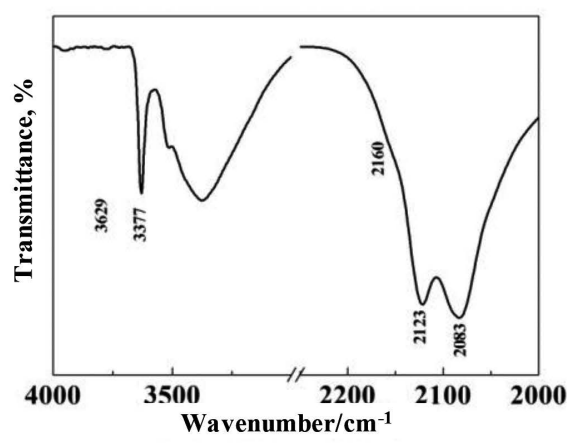

(A) SEM image (scale bar of $500 \mathrm{~nm}$ ); (B) EDS were different valence states of $\mathrm{Fe}$ and $\mathrm{Co}$ in the Co-Fe PBA NCs (Fig. 1D).

Catalytic activity of Co-Fe PBA NCs for the luminol CL system On the paper-based chip, the CL behavior of Co-Fe PBA NCs in the luminol-system was investigated. With Co-Fe PBA NCs added on the paper surface firstly, strong CL emission was obtained after the injection of luminol on the same paper detection zone (Fig. 2A). In comparison to the CL emission without Co-Fe PBA NCs, an excellent catalytic performance of Co-Fe PBA NCs in CL system was obtained. As we all know, $\mathrm{K}_{3}\left[\mathrm{Fe}(\mathrm{SCN})_{6}\right]$ is a commonly used oxidant in the luminol $\mathrm{CL}$ system and strong CL emission can always be obtained in the $\mathrm{K}_{3}\left[\mathrm{Fe}(\mathrm{SCN})_{6}\right]$-luminol system. Because $\mathrm{K}_{3}\left[\mathrm{Fe}(\mathrm{SCN})_{6}\right]$ was the reactant to synthesize $\mathrm{Co}-\mathrm{Fe} \mathrm{PBA} \mathrm{NCs}$, the influence of
$\mathrm{K}_{3}\left[\mathrm{Fe}(\mathrm{SCN})_{6}\right]$ should be first investigated. That is to say, $\mathrm{Co}-\mathrm{Fe}$ PBA NCs rather than $\mathrm{K}_{3}\left[\mathrm{Fe}(\mathrm{SCN})_{6}\right]$ can have a catalytic effect in this luminol CL system. For doing this, the synthesized CoFe PBA NCs was washed several times and the washing solution was collected for $\mathrm{CL}$ detection. The results are shown in Fig. 2B. From the results we can see that after Co-Fe PBA NCs was washed for three times, the CL signal was almost the same as the blank signal. So in this work, Co-Fe PBA NCs catalyzed the luminol CL system. The CL signal was recorded with Co-Fe PBA NCs compared to $\mathrm{Co}^{2+}$ or $\mathrm{Fe}(\mathrm{SCN})_{6}^{3-}$ in Fig. $2 \mathrm{C}$. As a result, Co-Fe PBA NCs exhibited a superior performance towards luminol CL. At the same time, when CA was added to the luminol-Co-Fe PBA NCs system, the CL intensity was significantly inhibited (Fig. 2C). 

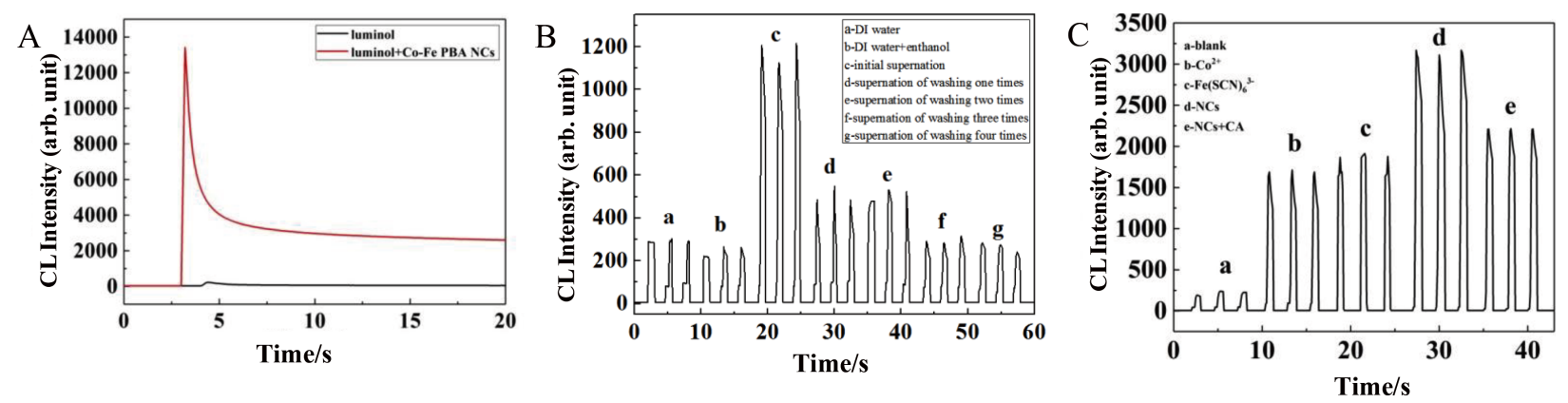

Fig. 2 (A) CL response curves of Co-Fe PBA NCs catalyzed luminol. Experimental conditions: luminol, $1.4 \mathrm{mM}$; Co-Fe PBA NCs, $2.5 \mathrm{mg} \mathrm{mL}^{-1}$. (B) CL intensity of Co-Fe PBA NCs supernatant with luminol after different washing times. Experimental conditions: luminol, $1.4 \mathrm{mM}$; Co-Fe PBA $\mathrm{NCs}, 2.5 \mathrm{mg} / \mathrm{mL}$. (C) CL intensity of luminol with $\mathrm{Fe}(\mathrm{SCN})_{6}{ }^{3-}, \mathrm{Co}^{2+}$, Co-Fe PBA NCs, and Co-Fe PBA NCs-CA. Experimental conditions: $\mathrm{Co}^{2+}, 0.06 \mathrm{mg} \mathrm{mL}^{-1}$; $\mathrm{Fe}(\mathrm{SCN})_{6}^{3-}, 0.4 \mathrm{mg} \mathrm{mL}^{-1}$; Co-Fe PBA NCs, $0.2 \mathrm{mg} \mathrm{mL}^{-1}$; CA concentration, $100 \mathrm{ng} \mathrm{mL}^{-1}$.
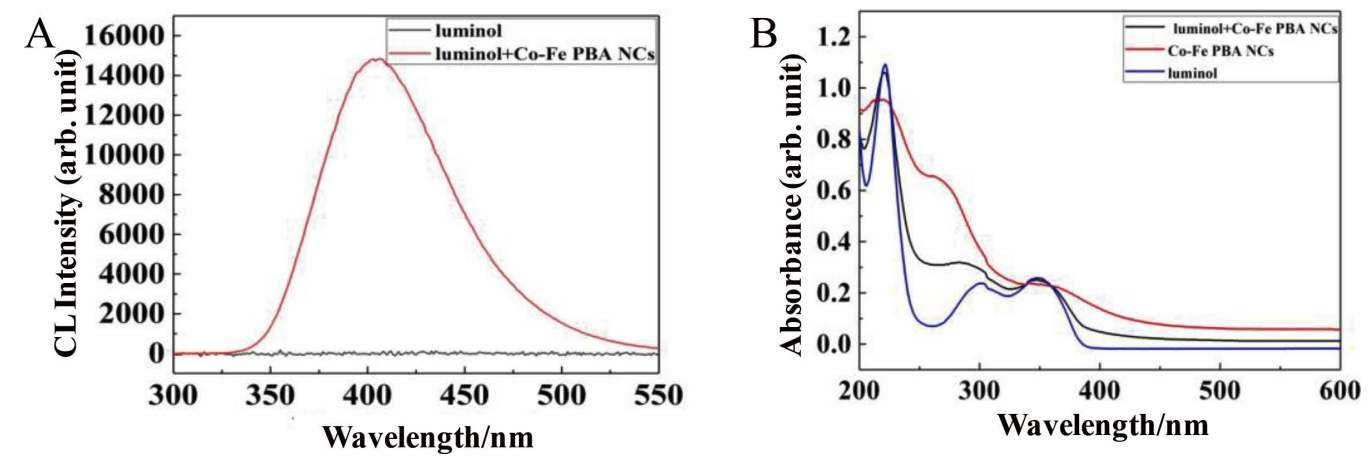

Fig. 3 (A) CL spectra of luminol-Co-Fe PBA NCs (red line). Experimental conditions: Co-Fe PBA NCs, $2.5 \mathrm{mg} \mathrm{mL}^{-1}$; luminol, $1.4 \mathrm{mM}$. (B) UV-Vis absorbance spectra of Co-Fe PBA NCs (red line), luminol (blue line), luminol-Co-Fe PBA NCs (black line). Experimental conditions: Co-Fe PBA NCs, $10^{-5} \mathrm{mg} \mathrm{mL}^{-1}$; luminol, $10^{-5} \mathrm{M}$.

The CL mechanism with Co-Fe PBA NCs

In order to understand the properties of the luminol-Co-Fe PBA NCs system, the emitting species of the CL spectrum were studied. The maximum CL wavelength with Co-Fe PBA NCs was $425 \mathrm{~nm}$ (Fig. 3A). This revealed that the luminophor was used as the excited-state 3-aminophthalate anions (3-APA*). Therefore, Co-Fe PBA NCs as a reagent to enhance the CL signals. ${ }^{34}$ Then, the role of Co-Fe PBA NCs in this luminol-CoFe PBA NCs system was investigated by UV-visible absorption measurements. There was no appreciable change in the maximum absorption position of the luminol-Co-Fe PBA NCs compared to the individual luminol or Co-Fe PBA NCs (Fig. 3B). Furthermore, Co-Fe PBA NCs was a catalyst in this luminol-Co-Fe PBA NCs CL system.

It is common that most CL systems need the involvement of extra oxidants, such as $\mathrm{H}_{2} \mathrm{O}_{2}{ }^{22}$ and $\mathrm{KMnO}_{4}{ }^{20}$ However, in this work no extra oxidant was added into the luminol-Co-Fe PBA NCs system. We then deduced that dissolved oxygen was participated in this system. The effect of dissolved oxygen in the luminol-Co-Fe PBA NCs CL system was investigated in Supporting Information (SI). Also, different valence states of $\mathrm{Co}$ (III) existed in the Co-Fe PBA NCs. $\mathrm{Li}^{35}$ reported in the 2000 year that $\mathrm{Co}$ (III) can react with dissolved oxygen to produce oxygen radical. That is one way to certify the excellent catalytic property of Co-Fe PBA NCs.
In the dissolved oxygen luminol system, different oxygen radicals were always formed. These oxygen radicals were then verified by electron spin resonance (ESR) and radical scavengers in SI. Different radicals may be formed in the luminol-Co-Fe PBA NCs CL system, including the superoxide radical anion $\left(\mathrm{O}_{2}{ }^{--}\right)$, the singlet oxygen $\left({ }^{1} \mathrm{O}_{2}\right)$, and the hydroxyl radical $\left(\mathrm{OH}^{*}\right)$. Effective radical scavengers of ascorbic acid (AA), ${ }^{36}$ uperoxide dismutase (SOD), ${ }^{37}$ 2,5-dimethylfuran ${ }^{36}$ and thiourea ${ }^{36}$ were investigated. For targeted validation, ESR was a powerful method to quanitify shot-lived radicals. Different spin adducts were used to distinguish different oxygen radical. In this study, 5,5-dimethyl-1-pyrroline- $N$-oxide (DMPO) ${ }^{38}$ was applied to capture $\mathrm{O}_{2}{ }^{-}$and $\mathrm{OH}^{-}$. The 1:2:2:1 quartet characteristic peak of the typical DMPO-OH• adduct (Fig. 4A) and the 1:1:1:1 quartet characteristic peak of typical DMPO- $\mathrm{O}_{2}{ }^{--}$adduct (Fig. 4B) were observed. The results indicated that $\mathrm{O}_{2}{ }^{-}$and $\mathrm{OH}^{-}$were formed during the whole process. Tetramethyl 4-piperidine (TEMP) was an efficient detection agent of ${ }^{1} \mathrm{O}_{2}$. With the addition of TEMP, a strong 1:1:1 triplet signal of the TEMP nitroxide radical ${ }^{38}$ was yielded. This indicated that ${ }^{1} \mathrm{O}_{2}$ was involved in the luminol-Co-Fe PBA NCs system (Fig. 4C).

The possible mechanism of the luminol-Co-Fe PBA NCs system was concluded to be as follows: Firstly, superoxide radicals were formed under the dissolved oxygen. Then, the formed $\mathrm{O}_{2}{ }^{-}$was converted into ${ }^{1} \mathrm{O}_{2}$ and $\mathrm{H}_{2} \mathrm{O}_{2}$. $\mathrm{OH}^{\bullet}$ was produced 

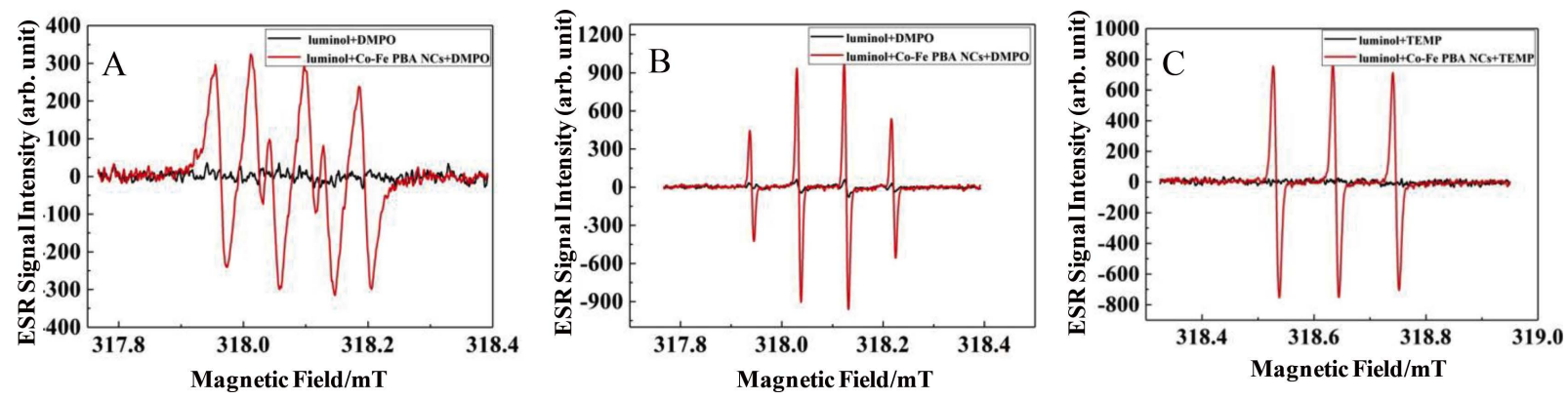

Fig. 4 ESR spectra of (A) $\mathrm{O}_{2}{ }^{-}$(B) $\mathrm{OH}^{\bullet}(\mathrm{C}){ }^{1} \mathrm{O}_{2}$ radicals in luminol (black line) and luminol-Co-Fe PBA NCs (red line) systems.

$$
\begin{aligned}
& \text { (1) } \mathrm{O}_{2} \stackrel{\mathrm{Co}-\mathrm{Fe} \text { PBA NCs }}{\longrightarrow} \mathrm{O}_{2}^{--} \\
& \text {(2) } \mathrm{O}_{2}^{--}+\mathrm{O}_{2}^{--}+2 \mathrm{H}_{2} \mathrm{O} \longrightarrow \mathrm{O}_{2}+\mathrm{H}_{2} \mathrm{O}_{2}+\mathrm{OH}^{-} \\
& \text {(3) } \mathrm{O}_{2}^{-\cdot}+\mathrm{H}_{2} \mathrm{O}_{2} \longrightarrow \mathrm{O}_{2}+\mathrm{OH}^{-}+\mathrm{OH}^{-} \\
& \text {(4) } \mathrm{L}+\mathrm{OH}^{-} \longrightarrow \mathrm{L}^{-}+\mathrm{H}_{2} \mathrm{O} \\
& \text { (5) } \mathrm{H}_{2} \mathrm{O}_{2}+\mathrm{OH}^{-} \longrightarrow \mathrm{HO}_{2}^{-}+\mathrm{H}_{2} \mathrm{O} \\
& \text { (6) } \mathrm{OH}^{-}+\mathrm{HO}_{2}^{-}+\mathrm{L}^{-} \longrightarrow \mathrm{O}_{2}^{--}+\mathrm{L}^{--}+\mathrm{H}_{2} \mathrm{O} \\
& \text { (7) } \mathrm{L}^{-}+\mathrm{O}_{2}^{--} \longrightarrow 3-\mathrm{APA}^{*} \\
& \text { 3-APA* } \longrightarrow 3-\mathrm{APA}+h v
\end{aligned}
$$

Scheme 2 The schematic mechanism of the luminol-Co-Fe PBA NCs system.

from $\mathrm{H}_{2} \mathrm{O}_{2}$ and $\mathrm{O}_{2}{ }^{-} . \mathrm{H}_{2} \mathrm{O}_{2}$ was converted into hydrogen peroxide ions $\left(\mathrm{HO}_{2}^{-}\right)$under the alkaline condition. Luminol molecules (L) transformed into luminol anions $\left(\mathrm{L}^{-}\right)$. Furthermore, $\mathrm{OH}^{-}$, $\mathrm{HO}_{2}^{-}$, and $\mathrm{L}^{-}$reacted and changed to the luminol anion radical $\left(\mathrm{L}^{-}\right)$and $\mathrm{O}_{2}^{--}$. At last, $\mathrm{O}_{2}^{--}$could react with $\mathrm{L}^{--}$to generate unstable excited-stated 3-aminophthalate anions (3-APA*) accompanying light emission at $425 \mathrm{~nm}$. The whole CL procedure is shown below in Scheme 2. CA is a kind of antioxidant that can capture and eliminate reactive oxygen species. As a result, after CA was added into the luminol-Co-Fe PBA NCs system, the CL intensity was inhibited.

\section{Optimization of experimental conditions}

To achieve a higher CL signal, the next conditions should be optimized in the luminol- $\mathrm{H}_{2} \mathrm{O}_{2}$ system on the paper-based chip: (A) $\mathrm{pH}$ value; (B) luminol concentration; (C) Co-Fe PBA NCs concentration in SI. At last, the best conditions of this CL system were a $\mathrm{pH}$ value of $11.5,1.4 \mathrm{mM}$ of luminol, and $2.5 \mathrm{mg} \mathrm{mL}^{-1}$ of Co-Fe PBA NCs.

\section{Sensitivity for caffeic acid}

The paper-based CL device was applied for CA measurements. Because CA can react with the active oxygen radicals, leading to the inhibition of the luminol-Co-Fe PBA NCs system, CA could be detected by the inhibited CL intensity. Under the optimum conditions, the CL inhibition intensity demonstrated an excellent linear relation with the CA concentration over the range of $10-800 \mathrm{ng} \mathrm{mL}^{-1}$ with a detection limit of $3 \mathrm{ng} \mathrm{mL}^{-1}$ based on $3 \sigma$ (Figs. 5A and 5B). In order to gain a better linearity, we adopted a piecewise linear. The linear relationship between $I$ and the concentration of $\mathrm{CA}$ in the range of $10-100$ and $100-800 \mathrm{ng} \mathrm{mL}^{-1}$, which can be expressed as $\Delta I=11.70 C+563.45\left(R^{2}=0.9970\right)$ and $\Delta I=2.3777 C+1507.8$ $\left(R^{2}=0.9987\right)$, respectively (Figs. 5C and 5D). What is more, some other methods for CA detection are summarized in Table 1. Compared to the other methods, the proposed method was simpler and had a relative lower detection limit for CA. Also, the proposed method can be used for real sample analysis with a low content of $\mathrm{CA}$.

\section{Selectivity}

In order to evaluate the selectivity of the proposed platform, the anti-interference capability against other coexisting substances were given in Fig. 6.

Some metal ions, anions, glucose, ascorbic acid, malic acid, tartaric acid, and citric acid ${ }^{39}$ were chosen here for the detection. Some interfering substances which have a similar structure to CA, like gallic acid, sinapic acid, and coumaric acid were also tested. When the concentrations of gallic acid, sinapic acid, coumaric acid, ascorbic acid, malic acid, tartaric acid, and citric acid were 20 times higher than $\mathrm{CA}$, there was no obvious influence on the detection of CA (Fig. 6A). Also, with the concentration of some metal ions, anions and glucose 100 times higher than $\mathrm{CA}$, no obvious inference occurred on the signal (Fig. 6B). Therefore, under a certain circumstance, the method has a good selectivity for the detection of CA.

\section{Determination of caffeic acid in real sample}

Tea polyphenols are the general name of polyphenol in tea, accounting for about $25 \%$ of its dry weight of tea. It is mainly composed of catechins, anthocyanins, flavonoids, and phenolic acids. The amount of phenolic acids accounts for about 10 $15 \%$ of tea polyphenols. CA is one of the phenolic acids. Actually, different PCs had been first tested with this luminol CL system. They were gallic acid, quercetin, catechin, and kaempferol. But the detection limits of these four PCs were much higher than CA. The sensitivity for CA is the best in this work. In order to evaluate the practical application of the CL paper-based platform, CA in green tea and black tea samples was detected. A certain amount of samples were extracted by ethanol, and diluted 300 times. The recovery values were obtained by adding different amounts of CA standard solutions into the two tea samples. The results are shown in Table 2. The recovery rates were between 98.4 and $105.3 \%$. This suggested that the method can be effectively applied in the practical analysis, not only in teas, but also in some wines, fruits, and vegetables. 

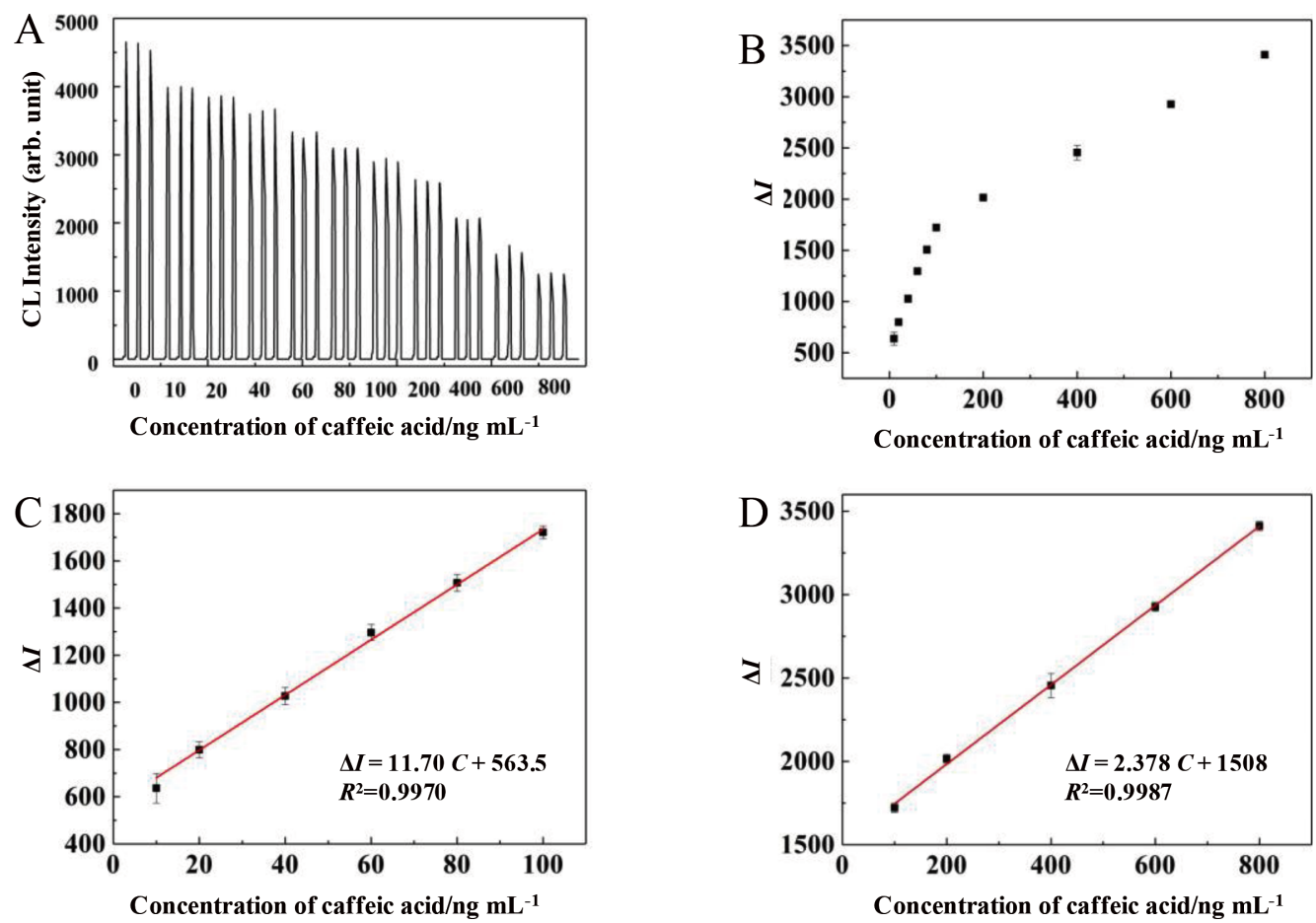

Fig. 5 Linear curves for the response of the $\mathrm{CL}$ intensity to different concentrations of CA based on the luminol-Co-Fe PBA NCs. Experimental conditions: pH, 11.5; luminol, $1.4 \mathrm{mM}$; Co-Fe PBA NCs, $2.5 \mathrm{mg} \mathrm{mL}^{-1}$ (error bar represents the standard deviation for three determinations).

Table 1 Different detection methods on CA in recent years

\begin{tabular}{llllr}
\hline \multicolumn{1}{c}{ Method } & \multicolumn{1}{c}{ Liner range } & \multicolumn{1}{c}{ Detection limit } & \multicolumn{1}{c}{ Sample } & Reference \\
\hline Fluorescence & $0.5-200 \mu \mathrm{M}$ & $0.11 \mu \mathrm{M}$ & Human plasma & 6 \\
UHPLC-ESI-MS/MS & $0.8800-880.0 \mathrm{ng} \mathrm{mL}^{-1}$ & Lower than $1.050 \mathrm{ng} \mathrm{mL}^{-1}$ & Plasma & 7 \\
Electrochemical & $0.5-60.0 \mu \mathrm{M}$ & $0.15 \mu \mathrm{M}$ & Wine & 8 \\
CZE & $2-100 \mathrm{mg} \mathrm{L}^{-1}$ & $0.5 \mathrm{mg} \mathrm{L}^{-1}$ & Wine & 9 \\
RP-HPLC-DAD & - & $1.1 \mathrm{mg} \mathrm{L}^{-1}$ & Wine & 10 \\
Chemiluminescence & $10-800 \mathrm{ng} \mathrm{mL}^{-1}$ & $3 \mathrm{ng} \mathrm{mL}^{-1}$ & Tea & This work \\
\hline
\end{tabular}
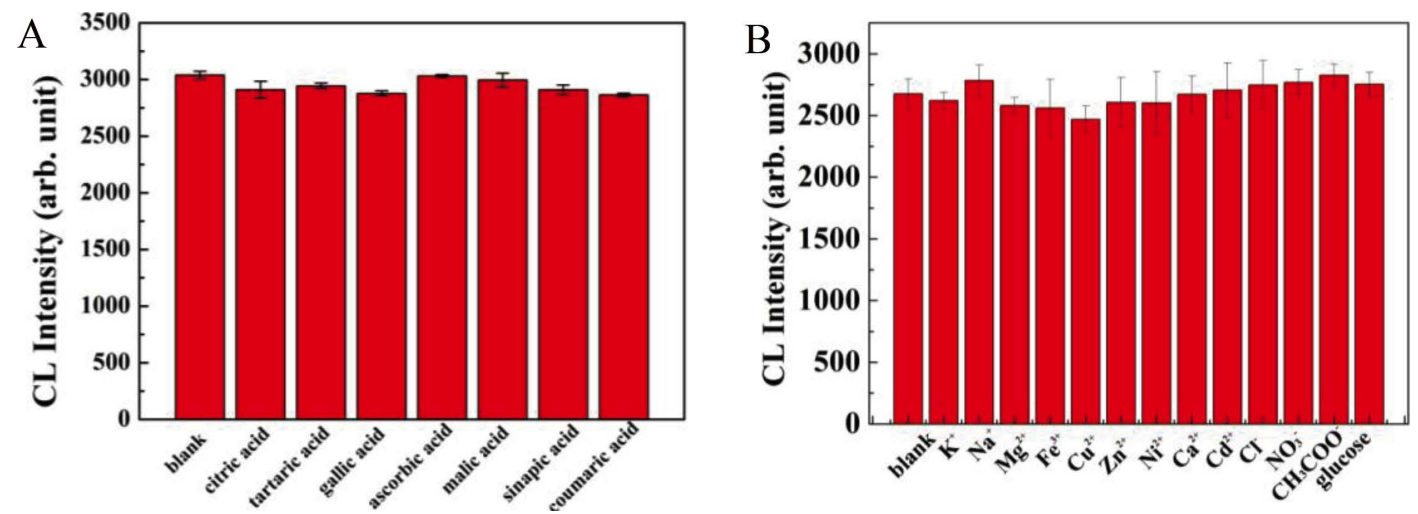

Fig. 6 Investigation on the interfering effects of other co-existing substances for the detection of CA. (A) The CL results with the coexisting substances concentration 20 times higher than CA. (B) The CL results with the coexisting substances concentration 100 times higher than CA. Experimental conditions: pH, 11.5; luminol, $1.4 \mathrm{mM}$; Co-Fe PBA NCs, $2.5 \mathrm{mg} \mathrm{mL}^{-1}$; CA, $100 \mathrm{ng} \mathrm{mL}^{-1}$ (error bar represents the standard deviation for three measurements). 
Table 2 Application of the CL method for the detection of CA in tea $(n=3)$

\begin{tabular}{cccccc}
\hline $\begin{array}{c}\text { Tea } \\
\text { sample }\end{array}$ & $\begin{array}{c}\text { Added/ } \\
\text { ng mL }^{-1}\end{array}$ & $\begin{array}{c}\text { Expected/ } \\
\text { ng mL }\end{array}$ & $\begin{array}{c}\text { Founded/ } \\
\text { ng mL }^{-1}\end{array}$ & $\begin{array}{c}\text { Recovery, } \\
\%\end{array}$ & $\begin{array}{c}\text { RSD, } \\
\%\end{array}$ \\
\hline Green tea & - & - & 18.9 & - & 4.2 \\
& 60.0 & 78.9 & 81.6 & 103.4 & 3.7 \\
& 80.0 & 98.9 & 104.1 & 105.3 & 1.3 \\
Black tea & 200.0 & 218.9 & 223.3 & 102.0 & 2.6 \\
& - & - & 17.6 & - & 2.8 \\
& 60.0 & 77.6 & 76.4 & 98.4 & 4.4 \\
& 200.0 & 97.6 & 100.5 & 103.1 & 6.5 \\
& 217.6 & 226.1 & 103.9 & 2.4 \\
\hline
\end{tabular}

\section{Conclusions}

In this work, a new luminol CL system with Co-Fe PBA NCs for the sensitive detection of CA on a paper-based chip is proposed. In the absence of additional oxidants, Co-Fe PBA NCs with oxidase-like activity can directly catalyze luminol to produce strong CL. The mechanism is that Co-Fe PBA NCs catalyze the formation of oxygen-containing free radicals in dissolved systems. CA can inhibit the CL for eliminating the reactive oxygen species of this system. On the paper-based chip, CA is sensitively determined in tea samples with a detection limit of $3 \mathrm{ng} \mathrm{mL}^{-1}$. This work can provide a new platform with paper-based chips for the detection of PCs detection in real samples.

\section{Conflict of Interest}

There are no conflicts to declare.

\section{Acknowledgements}

We are grateful to Shaanxi Province Science Foundation (No. 2018JM2002) for funding this work. The authors are also thankful for the Fundamental Research Funds for the Central Universities (No. GK201902009, No. GK201701002) and Program for Innovative Research Team in Shaanxi Province (No. 2014KCT-28) for supporting this work.

\section{Supporting Information}

The CL spectra of the luminol-Co-Fe PBA NCs solution under air-saturated and bubbled with $\mathrm{N}_{2}$. Effects of radical scavengers on the luminol-Co-Fe PBA NCs CL system. Effects of reactant conditions on luminol CL system in the presence of Co-Fe PBA NCs. This material is available free of charge on the Web at http://www.jsac.or.jp/analsci/.

\section{References}

1. B. Al Haddabi, H. A. Al Lawati, and F. O. Suliman, Food Chem., 2017, 214, 670.

2. L. P. Leong and G. Shui, Food Chem., 2002, 76, 69.

3. H. Filik, G. Cetintas, A. A. Avan, S. Aydar, S. N. Koc, and I. Boz, Talanta, 2013, 116, 245.

4. F. Shahidi and P. Ambigaipalan, J. Funct. Foods, 2015, 18, 820.
5. W. Wang, W. Sun, and L. Jin, Int. Immunopharm., 2017, 48, 61.

6. X. M. Xu, G. H. Xu, F. D. Wei, Y. Cen, M. L. Shi, X. Cheng, Y. Y. Chai, M. Sohail, and Q. Hu, J. Colloid Interface Sci., 2018, 529, 568.

7. Y. Du, Z. B. Wang, L. B. Wang, M. J. Gao, L. Q. Wang, C. L. Gan, and C. J. Yang, Molecules, 2017, 22, 1494.

8. F. R. F. Leite, W. d. J. R. Santos, and L. T. Kubota, Sens. Actuators, B, 2014, 193, 238.

9. H. Franquet-Griell, A. Checa, O. Nunez, J. Saurina, S. Hernandez-Cassou, and L. Puignou, J. Agric. Food Chem., 2012, 60, 8340.

10. R. Chagas, A. M. Lourenço, S. Monteiro, R. B. Ferreira, and L. M. Ferreira, Food Res. Int., 2017, 98, 103.

11. B. A. Mughairy, H. A. Al-Lawati, and F. O. Suliman, Sens. Actuators, B, 2018, 277, 517.

12. H. J. Liu, Y. Y. Su, D. Y. Deng, H. J. Song, and Y. Lv, Anal. Chem., 2019, 91, 9174.

13. Z. Wang, B. Dong, G. D. Feng, H. Y. Shen, Y. F. Huan, and Q. Fei, Anal. Sci., 2019, 35, 1135.

14. K. G. Wu and S. Q. Han, Anal. Sci., 2015, 31, 1249.

15. M. Luo, K. Shao, and Z. Long, Sens. Actuators, B, 2017, 240, 132.

16. J. Hassanzadeh, H. A. J. Al Lawati, and I. Al Lawati, Anal. Chem., 2019, 91, 10631.

17. J. Li, X. Zhao, L. J. Chen, H. L. Qian, W. L. Wang, C. Yang, and X. P. Yan, Anal. Chem., 2019, 91, 13191.

18. L. Choueir, V. S. Chedea, A. Calokerinos, and P. Kefalas, Food Chem., 2012, 133, 1039.

19. A. J. H. Al Lawati, B. Al Mughairy, I. Al Lawati, and F. O. Suliman, Luminescence, 2018, 33, 863.

20. B. Al Haddabi, H. A. J. Al Lawati, and F. O. Suliman, Talanta, 2016, 150, 399.

21. M. Iranifam and H. A. J. Al Lawati, Food Chem., 2019, 297, 124930

22. S. F. Li, S. F. Tao, F. F. Wang, J. G. Hong, and X. W. Wei, Microchim. Acta, 2010, 169, 73.

23. H. Chen, R. B. Li, H. F. Li, and J. M. Lin, J. Phys. Chem. $C, \mathbf{2 0 1 2}, 116,14796$.

24. B. R. Liu, X. H. Li, S. M. Liu, and X. Hun, Microchem. J., 2019, 145, 648.

25. W. B. Shi, X. D. Zhang, S. H. He, and Y. M. Huang, Chem. Commun., 2011, 47, 10785.

26. Y. W. Lu, X. D. Zhang, X. X. Mao, and Y. M. Huang, J. Mater. Chem. B, 2019, 7, 4661.

27. I. Lee, S. W. Han, and K. Kim, Chem. Commun., 2001, 18, 1782.

28. F. J. Ibanez and F. P. Zamborini, ACS Nano, 2008, 2, 1543.

29. J. W. Nai, B. Y. Guan, L. Yu, and X. W. Lou, Sci. Adv., 2017, 3, e1700732.

30. Y. Chen, X. Y. Guo, L. Zhang, and W. Liu, Microchim. Acta, 2019, 186, 112.

31. C. Duangdeewong, J. Sitanurak, P. Wilairat, D. Nacapricha, and S. Teerasong, Microchem. J., 2020, 152, 10447.

32. M. Zhao, H. F. Li, W. Liu, Y. M. Guo, and W. R. Chu, Biosens. Bioelectron., 2016, 79, 581.

33. D. J. Li, S. S. Zhang, X. Feng, H. J. Yang, F. Nie, and W. Y. Zhang, Sens. Actuators, B, 2019, 296, 126631.

34. B. X. Li, Z. J. Zhang, and M. L. Wu, Talanta, 2000, 51, 515

35. J. J. Du, L. Hao, Y. H. Li, and J. R. Lu, Anal. Chim. Acta, 2007, 582, 98.

36. L. Liu, C. Q. Sun, J. Yang, Y. Shi, Y. J. Long, and H. Z. Zheng, Chem. Eur. J., 2018, 24, 6148.

37. L. He, Z. W. Jiang, W. Li, C. M. Li, C. Z. Huang, and Y. F. $\mathrm{Li}$, ACS Appl. Mater. Interfaces, 2018, 10, 28868.

38. L. Gao, R. R. Yue, J. K. Xu, Z. Liu, and J. D. Chai, J. Electroanal. Chem., 2018, 816, 14. 